\title{
Decision Support System to Determine Qualified Corn using TOPSIS Method
}

\author{
Serly Dwy Lestary, Nongmaithem Ajith Singh, P. Nagaraja, K. Shankar, Wahidah Hashim, \\ Elena V. Smirnova, Andino Maseleno
}

\begin{abstract}
Corn is a staple food ingredient, one of the areas where people consume corn instead of rice is Southeast Sulawesi. Qualified corn is one of the superior seeds, while some types of superior corn are hybrid corn and composite corn. The TOPSIS method is one of the multi criteria decision-making methods with simple concepts and efficient competition and has the ability to measure relative performance and alternative decisions in mathematical form. To make it easier to find qualified corn, the Technique for Order preference method is used. Similarity to Ideal Solution. This is facilitating corn farmers to choose good and productive corn crop quality, applying the TOPSIS method (Technique for Order Preference by Similarity to Ideal Solution) in determining quality, to help provide alternatives in $m$ determine qualified corn plants. Based on the results of research conducted on 10 qualified corn alternatives that had been tested, a result is obtained: $V 1=0.577350 \mathrm{~V} 2=0.474992 \mathrm{V3}=0.510529 \mathrm{~V} 4=$ $0.463630 V 5=0.609622 V 6=0.435983 V 7=0.533048 V 8=$ $0.373977 \mathrm{~V} 9=0.530333 \mathrm{V10}=0.448275$ From the results it can be concluded that V5 $=0.609622$ had fulfilled the requirements and can be said to be good qualified corn.
\end{abstract}

Keywords : component; formatting; style; styling; insert.

\section{INTRODUCTION}

I Indonesia is a country with a very strategic geographical location, it is very beneficial for Indonesian citizen because almost all plants can be planted in Indonesia. Especially is corn crop, because corn plants have a very important role in order to increase the national food security in particular corn plant, corn has become a major commodity after Rice (Purwono et al., 2011). [1] For Indonesian people corn is a staple food ingredient. One of the areas that people consume corn as rice substitution is Sulawesi Tenggara. Planting with spacing aims to make the plant population to get the same parts of nutrients and sunlight. Planting distance is good used

Revised Manuscript Received on December 16, 2019

* Correspondence Author

Serly Dwy Lestary, Department of Information System, STMIK Pringsewu, Lampung, Indonesia

Nongmaithem Ajith Singh, Department of Computer Science, South East Manipur College. Email: manipurajithex@ gmail.com

*P. Nagaraja, School of Computing, Kalasalingam Academy of pnagaraja02@gmail.com, shankarcrypto@gmail.com

K. Shankar, School of Computing, Kalasalingam Academy of Research and Education, Krishnankoil, Tamilnadu, India. Email: shankarcrypto@gmail.com

Wahidah Hashim, School of Computing, Kalasalingam Academy of Research and Education, Krishnankoil, Tamilnadu, India.

Elena V. Smirnova, School of Computing, Kalasalingam Academy of Research and Education, Krishnankoil, Tamilnadu, India.

Andino Maseleno, School of Computing, Kalasalingam Academy of Research and Education, Krishnankoil, Tamilnadu, India. Research and Education, Krishnankoil, Tamilnadu, India. Emails:

in the corn plant is $50 \mathrm{~cm} \times 40 \mathrm{~cm}$ with 1 plant [2].

Qualified corn is one of the superior seeds, as for some kinds of superior corn one of them is hybrid corn, composite corn, corn that will be made as seedlings must be derived from the fertile corn and free of pests, corn derived from the fruit of corn must be dried from the tree, the corn whose flowers have been discarded, corn must also come from the corn that have big cogs, after that it can be said as good qualified corn, corn is a second important food ingredient after Rice. Besides being a staple food, corn is used for various kinds of processed including: vegetables, flour, ethanol, cooking oil, sugar, livestock feed, and industrial raw materials. [3]

Based on the previous research conducted by FridollinGratio. P. Raya Ola, (2013) stated that the data used in this research is primary data. Primary data obtained through field surveys. The results of previous research that the effect of planting distance to the production of corn 50x40 couldn't increase the production of corn cultivation, with the administration of 50x60 could give the best influence on the production of corn. [4] Based on previous research conducted by YudaPranata, HuripSantoso and Benjamin Widyamoko, obtained the results of research showing that the performance of corn products in Tanggamus District has not been efficient.[ 5]

The research is more interested to make observating using TOPSIS Method (Technique for Order Preference by Similarity to Ideal Solution) . Research further develops the existing problems and improve the system that is already running. Therefore, it requires special knowledge regarding corn quality, so it is needed a decision support system that is able to provide information and recommendations to the corn farmers about good qualified corn. Researchers will use the method TOPSIS (Technique for Order Preference by Similarity to Ideal Solution) in designing a decision support system.

This research needs problem limitations to match with what is already planned so that this research objective could be achieved. The limitations of the problem discussed in this research was using TOPSIS method, data sample that conducted for this research derived from interview or dialog to corn farmer so it would be very easy to get Information directly from the corn farmer.

\section{A. The Formulation of Problem}

As for the formulation of problems in this research are as follows:

\section{Published By:}


a. How to design a corn plant development system using TOPSIS method ((Technique for Order Preference by Similarity to Ideal Solution)

b. How to conduct rank to score weight that taken from variables that already conducted with using a method so the decisions taken is objective

c. How this system give alternative options that can be reference for consumers in selection of superior and qualified corn.

\section{B. Objective and Benefit}

The objectives for this research were:

a. To make it easier for corn farmers to choose good and productive corn crop quality.

b. To Apply the TOPSIS (Technique for Order Preference by Similarity to Ideal Solution) method in the determining quality.

To provide an alternative in determining qualified corn plants.

\section{THE FOUNDATION OF THEORY}

\section{A. Decision Support System}

According to Ahmad Abdul Chamid (2016), Decision support System is an interactive computer-based information system, by processing data with various models to solve unstructured problems that can provide information to be used by the Decision makers in making a decision. In a decision support system, an intellectual resource owned by a person is combined with computer capabilities to help improve the quality of the decisions taken. Decision making is a process of selecting an action among several alternatives, so the expected goal can be achieved. [6]

\section{B. Corn Corp}

Corn is one of the grain crops of the herbaceous family. This plant is one of the important food crops, in addition to wheat and rice. The corn plants originated in America that spread to Asia and Africa through business activities of European people to America. Around the 16th century Portugal spread to Asia including Indonesia. [7]

\section{Stages of Decisions Making}

According to Zulkifli (2016)stages that must that must be passed through decisions making process as follows[8]:

\section{Intelligence Stage}

This stage is the search process and the approach of problem scope and problem recognition process. Input data is obtained, processed, tested to identify the problem.

\section{Design Stage}

This stage is the process of developing and seeking alternative actions or solutions that can be taken is a simplified representation of real events, so the validation and verification process is required to know the accuracy model in researching existing problems.

\section{Selection Stage}

This stage is conducted to various alternative solutions that are raised at planning phase to be determined or to concern with criteria based on the objectives.

\section{Implementation Stage}

This stage is applied to the design of systems that have been made in the planning and implementation of alternative actions that have been selected at the selection stage..

\section{FMADM}

Fuzzy Multiple Attribute Decision Making (FMADM) is a method that used to search alternative from some alternative with predefined criteria. The essence of the FMADM method is to determine the score of the weights for each attribute, so then ranking process will select specified alternative. There are basically three approaches to find the score of attribute weights namely the subjective approach, the objective approach, and the integration approach between subjective and objective. Each approach has its strengths and weaknesses.

There are several methods that can be used to solve FMADM problems namely:

a. Simple Additive Weighting Method (SAW)

b. Weighted Product (WP)

c. ELECTRE

d. Technique for Order preference any Similarity to Ideal Solution (TOPSIS)

e. Analytic Hierarchy Process (AHP).[9]

\section{RESEARCH METHOD}

\section{A. Data Collection Stages}

1) Observation Methods

At this stage of observation researchers conducted a direct observation about corn plantation in the Tanggamus District, then it was continued with the process of scoring of corn that became the object of research. From the observation results the researchers found some corn from the alternative corn that had been determined which can be categorized as the best corn, by comparing the score of each alternative corn.

\section{2) Interview Method}

In this stage, researchers performed an approach to the community, especially corn farmers, which are used as research objects to obtain a data or information needed to help determine the judgment of good qualified corn in accordance with predefined criteria. By way of interviewing or interviews which then the results gained will be compared with the score of each other alternative.

\section{B. TOPSIS Method}

According to Tri Susilowati (2018), TOPSIS is one of the multi criteria or alternative choice decision making methods which is an alternative that has the smallest distance from the ideal positive solution and the largest distance from the ideal negative solution from a geometric point of view with using a distance of Euclidean. However, alternatives that have the smallest distance from an ideal positive solution, don't have to have the largest distance from the ideal negative solution. Therefore, TOPSIS consider both, the distance to ideal positive solution and the distance to the ideal negative 
solution simultaneously. The optimal solution in the TOPSIS method is obtained by determining the relative proximity of an alternative to the ideal positive solution. TOPSIS will rank the alternative based on the relative proximity value priority of an alternative to the ideal positive solution. The alternatives that have been ranked are used as a reference for decision makers to choose the best solution that is desired. The TOPSIS method is one of the Fuzzy Multiple Attribute Decision Making (FMADM) Method which is a method used to find the optimal alternatives of a number of alternatives with certain criteria [10].

The stages of this TOPSIS method are as follows:

1. TOPSIS starts by building a decision matrix, the $X$ decision matrix refers to the alternative which will be evaluated based on the criteria

$$
\boldsymbol{x}=\left(\begin{array}{c}
A_{1} X_{11} X_{12} X_{12} \ldots . X_{1 n} \\
A_{2} X_{11} X_{22} X_{23} \ldots . X_{2 n} \\
A_{3} X_{31} X_{32} X_{33} \ldots . X_{3 n} \\
A_{m} X_{m 1} X_{m 2} X X_{m 3} \ldots . X_{m n}
\end{array}\right)
$$

where $A_{i}(i=1,2,3, \ldots . m)$ is the possible alternative, $X_{j}$ $(j=1,2,3, \ldots . n)$ is attribute where an alternate performance is measured, $\mathrm{X}_{\mathrm{ij}}$ is an alternate $\mathrm{Ai}$ performance with the reference to the $X_{j}$ attribute.

2. Create normalized matrix decision

$$
\sqrt[r i j]{\sum_{i=1}^{m} x_{i}^{2} j}
$$

with $\mathrm{i}=1,2, \ldots, \mathrm{m}$

$\mathrm{j}=1,2, \ldots, \mathrm{n}$

where,

$$
\begin{gathered}
r_{i j}=\text { normalized matrix }[\mathrm{i}][\mathrm{j}] \\
\mathrm{X}_{\mathrm{ij}}=\text { decision matrix }[\mathrm{i}][\mathrm{j}]
\end{gathered}
$$

3. Create normalized, weighted matrix decision $\mathrm{V}_{\mathrm{ij}}=\mathrm{w}_{\mathrm{i}} \mathrm{r}_{\mathrm{ij}}$; with $\mathrm{i}=1,2, \ldots, \mathrm{m}$; and $\mathrm{j}=1,2, \ldots, \mathrm{n}$.

where,

$\mathrm{V}_{\mathrm{ij}}=$ Elements of a weighted normalized decision matrix $\mathrm{V}$

$\mathrm{w}_{\mathrm{i}}=\mathrm{j}^{\text {th }}$ criteria weight

$r_{i j}=$ Normalized decision matrix elements $R$

4. Ideal positive solution $\mathrm{A}+$ and ideal negative solution $\mathrm{A}$ can be determined based on normalized weight rating $\left(\mathrm{y}_{\mathrm{ij}}\right)$ as:

$$
\begin{aligned}
& A^{+}=\left(y_{1}^{+}, y_{2}^{+}, \ldots, y_{n}^{+},\right) ; \\
& A^{-}=\left(y_{1}^{-}, y_{2}^{-}, \ldots, y_{n}^{-},\right) ;
\end{aligned}
$$

where,

$$
V_{j}^{+}=\max Y_{i j} \mathrm{j} \text { is benefit attribute } \operatorname{Min} Y_{i j} \text { if } \mathrm{j} \text { is cost }
$$
attribute

$V_{j}^{-}=\min y i j$, if $\mathrm{j}$ is benefit attribute max yij, if $\mathrm{j}$ is cost attribute

5. Distance between Ai alternative and ideal positive solutions:

where,

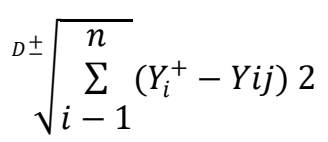

$D_{i}^{+}=$Distance between $\mathrm{A}_{\mathrm{i}}$ alternative and ideal positive solutions

$Y_{j}^{+}=$Ideal positive solutions [i]

$\mathrm{Y}_{\mathrm{ij}}=$ normalized matrix [i][j] $\mathrm{i}=1,2, \ldots \mathrm{m}$

where,

$D_{i}^{-}=$Distance $\mathrm{A}_{\mathrm{i}}$ alternative with ideal negative solutions

$Y_{j}^{-}=$ideal negative solution [i]

Yij $=$ normalized matrix $[\mathrm{i}][\mathrm{j}]$

6. The preference score for each alternative (Vi) is given as:

$$
\begin{aligned}
V_{i} & =\frac{D_{i}^{-}}{D_{i}^{-}+D_{i}^{+}} \\
\mathrm{i} & =1,2, \ldots \mathrm{m}
\end{aligned}
$$

$\mathrm{Vi}=$ Proximity of each alternative to the ideal solution

$D_{i}^{+}=$Distance between Ai alternative and ideal positive solutions

$D_{i}^{-}=$Distance between Ai alternative and ideal negative solutions

A larger score of $\mathrm{Vi}$ indicates that an alternative $\mathrm{Ai}$ is preferred.

\section{Criteria and Weight}

In this research it needs the criteria that will be calculated in determining the best and qualified corn for the farmers so the higher water content the better the corn gained, the lower water content it will produce not good corn. The ranking table used for each criterion is as below table.

Table-I: Alternative score for every criterion.

\begin{tabular}{|c|c|c|}
\hline Code criteria & Criteria & Score \\
\hline C1 & Water content & $10 \%$ \\
\hline C2 & Deformed Corn seed & $15 \%$ \\
\hline C3 & Corn waste & $15 \%$ \\
\hline C4 & Corn color & $15 \%$ \\
\hline C5 & Corn species & $10 \%$ \\
\hline C6 & Number of grains & $15 \%$ \\
\hline C7 & Corn Cob & $20 \%$ \\
\hline \multicolumn{3}{|c|}{ Total } \\
\hline
\end{tabular}

Table-II: Score

\begin{tabular}{|c|c|}
\hline Score & Description \\
\hline 1 & Very bad \\
\hline 2 & Bad \\
\hline 3 & Good enough \\
\hline 4 & Good \\
\hline 5 & Very good \\
\hline
\end{tabular}

Table-III: Deformed corn seed weight score(C2)

\begin{tabular}{|c|c|c|}
\hline Deformed corn seed & Score & Weight \\
\hline Because of disease & 2 & Bad \\
\hline Because of worm & 3 & Good enough \\
\hline
\end{tabular}

Table -IV: Corn waste weight score(C3)

\begin{tabular}{|c|c|c|}
\hline Corn waste & Score & Weight \\
\hline Straw & 3 & Good enough \\
\hline Cob & 2 & Bad \\
\hline Klobot & 4 & Good \\
\hline Corn skin & 1 & Very bad \\
\hline
\end{tabular}

Table-V: Corn color weight score (C4)

Published By: 


\begin{tabular}{|c|c|c|}
\hline Corn color & score & Weight \\
\hline Yellow & 4 & Good \\
\hline Orange & 5 & Very good \\
\hline White & 2 & Bad \\
\hline
\end{tabular}

Table-VI: Corn species weight score(C5)

\begin{tabular}{|c|c|c|}
\hline Corn species & score & Weight \\
\hline Sweet corn & 3 & Good enough \\
\hline Pearl corn & 2 & Bad \\
\hline Local corn & 3 & Good enough \\
\hline
\end{tabular}

Table-VII. Number of grains weight score (C6)

\begin{tabular}{|c|c|c|}
\hline $\begin{array}{c}\text { Number of } \\
\text { Grains }\end{array}$ & Score & Weight \\
\hline $\begin{array}{c}\text { Per line/cob max } \\
35 \text { grains }\end{array}$ & 4 & Good \\
\hline $\begin{array}{c}\text { Per line/cob min } \\
15-20 \text { grains }\end{array}$ & 3 & Good enough \\
\hline $\begin{array}{c}\text { Per seed/cob 560 } \\
\text { grains }\end{array}$ & 4 & Good \\
\hline
\end{tabular}

Table-VIII. Corn cob weight score (C7)

\begin{tabular}{|c|c|c|}
\hline Corn cob & score & weight \\
\hline Corn fruit & 3 & Good enough \\
\hline Corn skin & 2 & Bad \\
\hline
\end{tabular}

\section{Research Framework}

Framework in this research explains about how to select or choose qualified corn for farmers using TOPSIS method. This identification conducted by assessment form then the data collection process, and then next process is selection of qualified corn support system. In designing the decision support system of this qualified corn, a first analysis is conducted as well as to collect data from the farmers so that we know that in the selection of this qualified corn there are criteria and weight score that can prove qualified corn. Fig. 1 shows the description using a flowchart.

1. In this research is conducted identification of the objects of some corn that will be used as a research object.

2. Determined the criteria according to the title of our research using the TOPSIS method. 3. To make it easier for us to do research that we are encouraged to collect data according to what we want to be thorough.

4. In this stage we have entered in the discussion where we calculate or determine the criteria of each alternative. 5. Perform calculation manually starting with the calculations via Ms. Excel to make it easier and for the results to be the same as the calculations we do manually.

6. After determining the calculation of criteria of each alternative of course we will be able to find the best results for qualified corn.

7. Once finished will be re-checked by trying or testing it.

\section{EXPLANATION}

\section{A. Manual Test}

Alternative Table of all criteria is needed in the research obtained from the results of analysts of several alternates and existing criteria, so that it is obtained the matrix of the weighted decision to perform the calculation manually or computerized.

After the weights for the criteria obtained, the next process is carried out rank by using the TOPSIS method stages performed on the TOPSIS method are as follows:

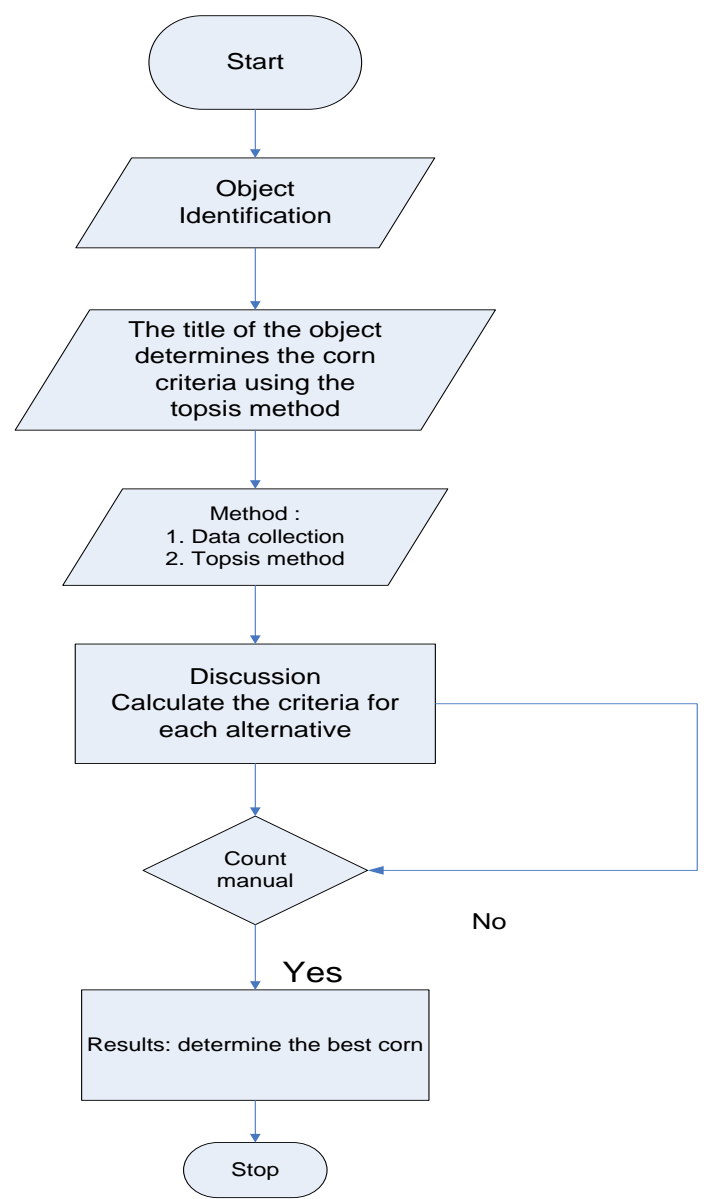

Fig 1. Flowchart Diagram

Table-IX: Decisions Matrix

\begin{tabular}{|c|c|c|c|c|c|c|c|}
\hline Alternative & C1 & C2 & C3 & C4 & C5 & C6 & C7 \\
\hline A1 & 3 & 4 & 1 & 3 & 3 & 2 & 3 \\
\hline A2 & 3 & 3 & 1 & 4 & 3 & 1 & 2 \\
\hline A3 & 1 & 2 & 3 & 2 & 2 & 4 & 2 \\
\hline A4 & 1 & 1 & 3 & 3 & 1 & 2 & 3 \\
\hline A5 & 4 & 2 & 4 & 4 & 4 & 2 & 2 \\
\hline A6 & 3 & 2 & 4 & 1 & 1 & 2 & 2 \\
\hline A7 & 4 & 4 & 3 & 2 & 2 & 3 & 1 \\
\hline A8 & 2 & 1 & 2 & 2 & 1 & 1 & 3 \\
\hline A9 & 2 & 3 & 1 & 3 & 2 & 4 & 2 \\
\hline A10 & 2 & 2 & 3 & 2 & 3 & 2 & 2 \\
\hline
\end{tabular}

Create normalized matrix decision

$$
\begin{gathered}
\left(X_{1}\right)=\sqrt{3^{2}+3^{2} 1^{2}+1^{2}+4^{2}+3^{2}+4^{2}+2^{2}+2^{2}+2^{2}} \\
=8,544003 \\
r_{11}=\frac{X_{11}}{\left|X_{1}\right|}=\frac{3}{8,544003}=0,351123 \\
r_{21}=\frac{X_{21}}{\left|X_{1}\right|}=\frac{3}{8,544003}=0,351123
\end{gathered}
$$

Published By: 


$$
\begin{aligned}
& r_{31}=\frac{X_{31}}{\left|X_{1}\right|}=\frac{1}{8,544003}=0,117041 \\
& r_{41}=\frac{X_{41}}{\left|X_{1}\right|}=\frac{1}{8,544003}=0,117041 \\
& r_{51}=\frac{X_{51}}{\left|X_{1}\right|}=\frac{4}{8,544003}=0,351123 \\
& r_{61}=\frac{X_{61}}{\left|X_{1}\right|}=\frac{3}{8,544003}=0,351123 \\
& r_{71}=\frac{X_{71}}{\left|X_{1}\right|}=\frac{4}{8,544003}=0,468164 \\
& r_{81}=\frac{X_{81}}{\left|X_{1}\right|}=\frac{2}{8,544003}=0,234082 \\
& r_{91}=\frac{X_{91}}{\left|X_{1}\right|}=\frac{2}{8,544003}=0,234082 \\
& r_{101}=\frac{X_{10}}{\left|X_{1}\right|}=\frac{2}{8,544003}=0,234082
\end{aligned}
$$$$
\left(X_{2}\right)=\sqrt{4^{2}+3^{2}+2^{2}+1^{2}+2^{2}+2^{2}+4^{2}+1^{2}+3^{2}+2^{2}}
$$$$
=8,246211
$$$$
r_{12}=\frac{X_{12}}{\left|X_{2}\right|}=\frac{4}{8,246211}=0,485071
$$$$
r_{22}=\frac{X_{22}}{\left|X_{2}\right|}=\frac{3}{8,246211}=0,363803
$$$$
r_{32}=\frac{X_{32}}{\left|X_{2}\right|}=\frac{2}{8,246211}=0,242535
$$$$
r_{42}=\frac{X_{42}}{\left|X_{2}\right|}=\frac{1}{8,246211}=0,121267
$$$$
r_{52}=\frac{X_{52}}{\left|X_{2}\right|}=\frac{2}{8,246211}=0,242535
$$$$
r_{62}=\frac{X_{62}}{\left|X_{2}\right|}=\frac{2}{8,246211}=0,242535
$$$$
r_{72}=\frac{X_{72}}{\left|X_{2}\right|}=\frac{4}{8,246211}=0,485071
$$$$
r_{82}=\frac{X_{82}}{\left|X_{2}\right|}=\frac{1}{8,246211}=0,121267
$$$$
r_{92}=\frac{X_{92}}{\left|X_{2}\right|}=\frac{3}{8,246211}=0,363803
$$$$
r_{102}=\frac{X_{102}}{\left|X_{2}\right|}=\frac{2}{8,246211}=0,242535
$$$$
\left(X_{3}\right)=\sqrt{1^{2}+1^{2} 3^{2}+3^{2}+4^{2}+4^{2}+3^{2}+2^{2}+1^{2}+3^{2}}
$$$$
=8,660254
$$$$
r_{13}=\frac{X_{13}}{\left|X_{3}\right|}=\frac{1}{8,660254}=0,115470
$$$$
r_{23}=\frac{X_{23}}{\left|X_{3}\right|}=\frac{1}{8,660254}=0,115470
$$$$
r_{33}=\frac{X_{33}}{\left|X_{3}\right|}=\frac{3}{8,660254}=0,346410
$$$$
r_{43}=\frac{X_{43}}{\left|X_{3}\right|}=\frac{3}{8,660254}=0,346410
$$$$
r_{53}=\frac{X_{53}}{\left|X_{3}\right|}=\frac{4}{8,660254}=0,461880
$$$$
r_{63}=\frac{X_{63}}{\left|X_{3}\right|}=\frac{4}{8,660254}=0,461880
$$$$
r_{73}=\frac{X_{73}}{\left|X_{3}\right|}=\frac{3}{8,660254}=0,346410
$$$$
r_{83}=\frac{X_{83}}{\left|X_{3}\right|}=\frac{2}{8,660254}=0,230940
$$$$
r_{93}=\frac{X_{93}}{\left|X_{3}\right|}=\frac{1}{8,660254}=0,115470
$$$$
r_{103}=\frac{X_{103}}{\left|X_{3}\right|}=\frac{3}{8,660254}=0,346410
$$$$
\left(X_{4}\right)=\sqrt{3^{2}+4^{2} 2^{2}+3^{2}+4^{2}+1^{2}+2^{2}+2^{2}+3^{2}+2^{2}}
$$

$$
=8,717797
$$

$r_{14}=\frac{X_{14}}{\left|X_{4}\right|}=\frac{3}{8,717797}=0,344123$

$r_{24}=\frac{X_{24}}{\left|X_{4}\right|}=\frac{4}{8,717797}=0,458831$

$r_{34}=\frac{X_{34}}{\left|X_{4}\right|}=\frac{2}{8,717797}=0,229415$

$r_{44}=\frac{X_{44}}{\left|X_{4}\right|}=\frac{3}{8,717797}=0,344123$

$r_{54}=\frac{X_{54}}{\left|X_{4}\right|}=\frac{4}{8,717797}=0,458831$

$r_{64}=\frac{X_{64}}{\left|X_{4}\right|}=\frac{1}{8,717797}=0,114707$

$r_{74}=\frac{X_{74}}{\left|X_{4}\right|}=\frac{2}{8,717797}=0,229415$

$r_{84}=\frac{X_{84}}{\left|X_{4}\right|}=\frac{2}{8,717797}=0,229415$

$$
\begin{aligned}
& r_{94}=\frac{X_{94}}{\left|X_{4}\right|}=\frac{3}{8,717797}=0,344123 \\
& r_{104}=\frac{X_{104}}{\left|X_{4}\right|}=\frac{2}{8,717797}=0,229415 \\
& \left(X_{5}\right)=\sqrt{3^{2}+3^{2} 2^{2}+1^{2}+4^{2}+1^{2}+2^{2}+1^{2}+2^{2}+3^{2}} \\
& =7,615773 \\
& r_{15}=\frac{X_{15}}{\left|X_{5}\right|}=\frac{3}{7,615773}=0,393919 \\
& r_{25}=\frac{X_{25}}{\left|X_{5}\right|}=\frac{3}{7,615773}=0,393919 \\
& r_{35}=\frac{X_{35}}{\left|X_{5}\right|}=\frac{2}{7,615773}=0,262612 \\
& r_{45}=\frac{X_{45}}{\left|X_{5}\right|}=\frac{1}{7,615773}=0,131306 \\
& r_{55}=\frac{X_{55}}{\left|X_{5}\right|}=\frac{4}{7,615773}=0,525225 \\
& r_{65}=\frac{X_{65}}{\left|X_{5}\right|}=\frac{1}{7,615773}=0,131306 \\
& r_{75}=\frac{X_{75}}{\left|X_{5}\right|}=\frac{2}{7,615773}=0,262612 \\
& r_{85}=\frac{X_{85}}{\left|X_{5}\right|}=\frac{1}{7,615773}=0,131306 \\
& r_{95}=\frac{X_{95}}{\left|X_{5}\right|}=\frac{2}{7,615773}=0,262612 \\
& r_{105}=\frac{X_{105}}{\left|X_{5}\right|}=\frac{3}{7,615773}=0,393919 \\
& \left(X_{6}\right)=\sqrt{2^{2}+1^{2} 4^{2}+2^{2}+2^{2}+2^{2}+3^{2}+1^{2}+4^{2}+2^{2}} \\
& =7,937253 \\
& r_{16}=\frac{X_{16}}{\left|X_{6}\right|}=\frac{2}{7,937253}=0,251976 \\
& r_{26}=\frac{X_{26}}{\left|X_{6}\right|}=\frac{1}{7,937253}=0,125988 \\
& r_{36}=\frac{X_{36}}{\left|X_{6}\right|}=\frac{4}{7,937253}=0,503953 \\
& r_{46}=\frac{X_{46}}{\left|X_{6}\right|}=\frac{2}{7,937253}=0,251976 \\
& r_{56}=\frac{X_{56}}{\left|X_{6}\right|}=\frac{2}{7,937253}=0,251976 \\
& r_{66}=\frac{X_{66}}{\left|X_{6}\right|}=\frac{2}{7,937253}=0,251976 \\
& r_{76}=\frac{X_{76}}{\left|X_{6}\right|}=\frac{3}{7,937253}=0,377964 \\
& r_{86}=\frac{X_{86}}{\left|X_{6}\right|}=\frac{1}{7,937253}=0,125988 \\
& r_{96}=\frac{X_{96}}{\left|X_{6}\right|}=\frac{4}{7,937253}=0,503953 \\
& r_{106}=\frac{X_{106}}{\left|X_{6}\right|}=\frac{2}{7,937253}=0,251976 \\
& \left(X_{7}\right)=\sqrt{3^{2}+2^{2} 2^{2}+3^{2}+2^{2}+2^{2}+1^{2}+3^{2}+2^{2}+2^{2}} \\
& =7,211102 \\
& r_{17}=\frac{X_{17}}{\left|X_{7}\right|}=\frac{3}{7,211102}=0,416025 \\
& r_{27}=\frac{X_{27}}{\left|X_{7}\right|}=\frac{2}{7,211102}=0,277350 \\
& r_{37}=\frac{X_{37}}{\left|X_{7}\right|}=\frac{2}{7,211102}=0,277350 \\
& r_{47}=\frac{X_{47}}{\left|X_{7}\right|}=\frac{3}{7,211102}=0,416025 \\
& r_{57}=\frac{X_{57}}{\left|X_{7}\right|}=\frac{2}{7,211102}=0,277350 \\
& r_{67}=\frac{X_{67}}{\left|X_{7}\right|}=\frac{2}{7,211102}=0,277350 \\
& r_{77}=\frac{X_{77}}{\left|X_{7}\right|}=\frac{1}{7,211102}=0,138675 \\
& r_{87}=\frac{X_{87}}{\left|X_{7}\right|}=\frac{3}{7,211102}=0,416025 \\
& r_{97}=\frac{X_{97}}{\left|X_{7}\right|}=\frac{2}{7,211102}=0,277350 \\
& r_{107}=\frac{X_{107}}{\left|X_{7}\right|}=\frac{2}{7,211102}=0,277350
\end{aligned}
$$

Published By: 

(R):

$R=\left(\begin{array}{cccc}0,351123 & 0,4850710,115470 & 0,344123 & 0,393919 \\ 0,351123 & 0,3638030,115470 & 0,458831 & 0,393919 \\ 0,1170410,2425360,346410 & 0,229415 & 0,262612 \\ 0,1170410,1212680,346410 & 0,344123 & 0,131306 \\ 0,351123 & 0,2425360,461880 & 0,458831 & 0,525225 \\ 0,3511230,2425350,461880 & 0,114707 & 0,131306 \\ 0,468165 & 0,4850710,346410 & 0,229415 & 0,262612 \\ 0,234082 & 0,1212670,230940 & 0,229415 & 0,131306 \\ 0,234082 & 0,3638030,115470 & 0,344123 & 0,262612 \\ 0,234082 & 0,2425350,346410 & 0,229415 & 0,393919\end{array}\right)$

$$
R=\left(\begin{array}{llll}
0,351123 & 0,4850710,115470 & 0,344123 & 0,393919 \\
0,3511230,3638030,115470 & 0,458831 & 0,393919 \\
0,1170410,2425360,346410 & 0,229415 & 0,262612 \\
0,1170410,1212680,346410 & 0,344123 & 0,131306 \\
0,3511230,2425360,461880 & 0,458831 & 0,525225 \\
0,3511230,2425350,461880 & 0,114707 & 0,131306 \\
0,4681650,4850710,346410 & 0,229415 & 0,262612 \\
0,2340820,1212670,230940 & 0,229415 & 0,131306 \\
0,2340820,3638030,115470 & 0,344123 & 0,262612 \\
0,2340820,2425350,346410 & 0,229415 & 0,393919
\end{array}\right)
$$

After obtained normalized matrix (R), then calculate $\mathrm{V}$ matrix based on equation:

$$
\begin{aligned}
& V_{I J}=W_{J} X_{I J} \\
& V_{1}=W_{1} R_{11} \quad=(0,1)(0,351123) \\
& =0,035112 \\
& V_{12}=W_{2} R_{12}=(0,15)(0,485071) \\
& =0,072761 \\
& V_{I 3}=W_{3} R_{13}=(0,15)(0,115470) \\
& =0,017321 \\
& V_{14}=W_{4} R_{14}=(0,15)(0,344123) \\
& =0,051619 \\
& V_{I 5}=W_{5} R_{15}=(0,1)(0,393919) \\
& =0,039392 \\
& V_{I 6}=W_{6} R_{16} \quad=(0,15)(0,251976) \\
& =0,037796 \\
& V_{I 7}=W_{7} R_{17}=(0,20)(0,416025) \\
& =0,083205 \\
& V_{21}=W_{1} R_{21} \quad=(0,1)(0,351123) \\
& =0,035112 \\
& V_{22}=W_{2} R_{22}=(0,15)(0,363803) \\
& =0,054570 \\
& V_{23}=W_{3} R_{23}=(0,15)(0,115470) \\
& =0,0173205 \\
& V_{24}=W_{4} R_{24} \quad=(0,15)(0,458831) \\
& =0,068824 \\
& V_{25}=W_{5} R_{25} \quad=(0,1)(0,393919) \\
& =0,393919 \\
& V_{26}=W_{6} R_{27}=(0,15)(0,125988) \\
& =0,018898 \\
& V_{27}=W_{7} R_{27} \quad=(0,20)(0,277350) \\
& =0,055470 \\
& V_{31}=W_{1} R_{31} \quad=(0,1)(0,117041) \\
& =0,011704 \\
& V_{32}=W_{2} R_{31}=(0,15)(0,242535) \\
& =0,036380 \\
& V_{33}=W_{3} R_{33}=(0,15)(0,346410) \\
& =0,051961 \\
& V_{34}=W_{4} R_{34} \quad=(0,15)(0,229415) \\
& =0,034412
\end{aligned}
$$

From calculation results above obtained normalized matrix

$$
\begin{aligned}
& V_{35}=W_{5} R_{35}=(0,1)(0,262612) \\
& =0,262612 \\
& V_{36}=W_{6} R_{36}=(0,15)(0,503953) \\
& =0,075593 \\
& V_{37}=W_{7} R_{37}=(0,20)(0,277350) \\
& =0,055470 \\
& V_{41}=W_{1} R_{I 41}=(0,1)(0,117041) \\
& =0,0117041 \\
& V_{42}=W_{2} R_{42}=(0,15)(0,121267) \\
& =0,018190 \\
& V_{43}=W_{3} R_{43}=(0,15)(0,346410) \\
& =0,051961 \\
& V_{44}=W_{4} R_{44} \quad=(0,15)(0,344123) \\
& =0,051618 \\
& V_{45}=W_{5} R_{45} \quad=(0,1)(0,131306) \\
& =0,131306 \\
& V_{46}=W_{6} R_{46} \quad=(0,15)(0,251976) \\
& =0,037796 \\
& V_{47}=W_{7} R_{47}=(0,20)(0,416025) \\
& =0,083205 \\
& V_{51}=W_{1} R_{51}=(0,1)(0,468164) \\
& =0,046816 \\
& V_{52}=W_{2} R_{52}=(0,15)(0,242535) \\
& =0,036380 \\
& V_{53}=W_{3} R_{53}=(0,15)(0,461880) \\
& =0,069282 \\
& V_{54}=W_{4} R_{54}=(0,15)(0,458831) \\
& V_{55}=W_{5} R_{55} \quad=(0,1)(0,525225) \\
& =0052525 \\
& V_{56}=W_{6} R_{56}=(0,15)(0,251976) \\
& V_{57}=W_{7} R_{57}=(0,20)(0,277350) \\
& =0,055470 \\
& V_{61}=W_{1} R_{61}=(0,1)(0,351123) \\
& =0,035112 \\
& V_{62}=W_{2} R_{62} \quad=(0,15)(0,242535) \\
& =0,036380 \\
& V_{63}=W_{3} R_{63} \quad=(0,15)(0,461880) \\
& =0,069282 \\
& V_{64}=W_{4} R_{64}=(0,15)(0,114707) \\
& =0,017206 \\
& V_{65}=W_{5} R_{65} \quad=(0,1)(0,131306) \\
& =0,013130 \\
& V_{66}=W_{6} R_{66}=(0,15)(0,251976) \\
& =0,037796 \\
& V_{67}=W_{7} R_{67} \quad=(0,20)(0,277350) \\
& =0,055470 \\
& V_{71}=W_{1} R_{71}=(0,1)(0,468164) \\
& =0,046816 \\
& V_{72}=W_{2} R_{72}=(0,15)(0,485071) \\
& =0,072760 \\
& V_{73}=W_{3} R_{73}=(0,15)(0,346410) \\
& =0,051961 \\
& V_{74}=W_{4} R_{74} \quad=(0,15)(0,229415) \\
& =0,034412 \\
& V_{75}=W_{5} R_{75}=(0,1)(0,262612) \\
& V_{76}=W_{6} R_{76}=0,026261=(0,15)(0,377964) \\
& =0,056695 \\
& V_{77}=W_{7} R_{77} \quad= \\
& (0,20)(0,138675) \\
& =0,027735
\end{aligned}
$$




$$
\begin{aligned}
& \begin{array}{c}
V_{81}=W_{1} R_{81}=(0,1)(0,234082) \\
=0,023408
\end{array} \\
& V_{82}=W_{2} R_{82} \quad=(0,15)(0,121267) \\
& =0,018190 \\
& V_{83}=W_{3} R_{83} \quad=(0,15)(0,230940) \\
& =0,034641 \\
& V_{84}=W_{4} R_{84} \quad=(0,15)(0,229415) \\
& =0,034412 \\
& V_{85}=W_{5} R_{85} \quad=(0,1)(0,131306) \\
& =0,013130 \\
& V_{86}=W_{6} R_{86}=(0,15)(0,125988) \\
& =0,018898 \\
& V_{87}=W_{7} R_{87} \quad=(0,20)(0,416025) \\
& =0,083205 \\
& V_{91}=W_{1} R_{91} \quad=(0,1)(0,234082) \\
& =0,023408 \\
& V_{92}=W_{2} R_{92}=(0,15)(0,363803) \\
& =0,054570 \\
& V_{93}=W_{3} R_{93}=(0,15)(0,115470) \\
& =0,017320 \\
& V_{94}=W_{4} R_{94}=(0,15)(0,344123) \\
& =0,051618 \\
& V_{95}=W_{5} R_{95}=(0,1)(0,262612) \\
& =0,026261 \\
& V_{96}=W_{6} R_{96}=(0,15)(0,503935) \\
& =0,075591 \\
& V_{97}=W_{7} R_{97}=(0,20)(0,277350) \\
& =0,055470 \\
& V_{101}=W_{1} R_{101}=(0,1)(0,234082) \\
& =0,0234082 \\
& V_{102}=W_{2} R_{102}=(0,15)(0,242535) \\
& =0,036380 \\
& V_{103}=W_{3} R_{103}=(0,15)(0,346410) \\
& =0,051961 \\
& V_{104}=W_{4} R_{104}=(0,15)(0,229415) \\
& =0,034412 \\
& V_{105}=W_{5} R_{105}=(0,1)(0,393919) \\
& =0,039393 \\
& V_{106}=W_{6} R_{106}=(0,15)(0,251976) \\
& =0,037796 \\
& V_{107}=W_{7} R_{107}=(0,20)(0,277350) \\
& =0,055470
\end{aligned}
$$

From calculation above, obtained matrix Y

$$
Y=\left(\begin{array}{llll}
0,035112 & 0,0727610,017321 & 0,051619 & 0,039392 \\
0,035112 & 0,0545710,017321 & 0,0688250,039392 \\
0,011704 & 0,0363800,051962 & 0,0344120,026261 \\
0,011704 & 0,0181900,051962 & 0,0516190,013131 \\
0,035112 & 0,0363800,069282 & 0,0688250,052523 \\
0,035112 & 0,0363800,069282 & 0,0172060,013131 \\
0,046816 & 0,0727610,051962 & 0,0344120,026261 \\
0,023408 & 0,0181900,034641 & 0,0344120,013131 \\
0,023408 & 0,0545710,017321 & 0,0516190,026261 \\
0,023408 & 0,0363800,051962 & 0,0344120,039392
\end{array}\right)
$$

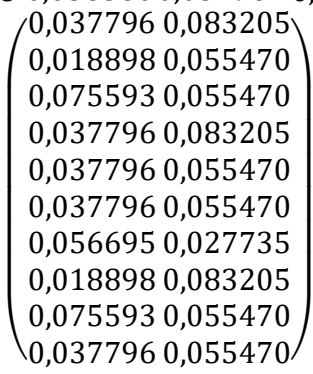

$(0,035112 ; 0,035112 ; 0,011704 ; 0,011704 ;$

$\left\{\begin{array}{c}0,035112 ; 0,035112 ; 0,046816 ; 0,023408 ; \\ 0,023408 ; 0,023408\end{array}\right\}$

$$
0,023408 ; 0,023408
$$$$
=0,046816
$$$$
y_{2}^{+}=\max
$$

$(0,072761 ; 0,054570 ; 0,036380 ; 0,018190 ;$

$\left\{\begin{array}{c}0,036380 ; 0,036380 ; 0,072760 ; 0,018190 ; \\ 0,054570 ; 0,036380\end{array}\right\}$

$$
\begin{aligned}
& =0,072761 \\
& y_{3}^{+}=\max
\end{aligned}
$$

$(0,017330 ; 0,017320 ; 0,051961 ; 0,051961 ;$

$\{0,069282 ; 0,069282 ; 0,051961 ; 0,034641 ;\}$

$$
\begin{gathered}
0,017320 ; 0,051961 \\
=0,069282 \\
y_{4}^{+}=\max
\end{gathered}
$$

$(0,051634 ; 0,068825 ; 0,034412 ; 0,051618$;

$\{0,068825 ; 0,017206 ; 0,034412 ; 0,034412 ;\}$

$$
\begin{gathered}
0,051618 ; 0,034412 \\
=0,068825
\end{gathered}
$$$$
y_{5}^{+}=\max
$$

$(0,039392 ; 0,039392 ; 0,026261 ; 0,013131 ;$

$\left\{\begin{array}{c}0,052523 ; 0,013131 ; 0,026261 ; 0,013131 ; \\ 0,026261 ; 0,039392\end{array}\right\}$ $=0,052523$

$$
y_{6}^{+}=\max
$$

$(0,037796 ; 0,018898 ; 0,075593 ; 0,037796$;

$\left\{\begin{array}{c}0,037796 ; 0,037796 ; 0,056695 ; 0,018898 ; \\ 0,075593 ; 0,037796\end{array}\right\}$

$$
=0,075593
$$$$
y_{7}^{+}=\max
$$

$(0,083205 ; 0,055470 ; 0,055470 ; 0,083205 ;$

$\left\{\begin{array}{c}0,055470 ; 0,055470 ; 0,027735 ; 0,083205 ; \\ 0,055470 ; 0,055470\end{array}\right\}$ $=0,083205$

$A^{+}=(0,046816 ; 0,072761 ; 0,069282 ; 0,068825 ; 0,052523$; $0,075593 ; 0,083205)$

Negative ideal solution calculated based on equation:

$$
\begin{gathered}
A^{-}=\left(y_{1}^{-}, y_{2}^{-} \ldots y_{n}^{-}\right) \\
y_{1}^{-}=\min
\end{gathered}
$$

0,$035112 ; 0,035112 ; 0,011704 ; 0,011703$;

$\{0,035112 ; 0,035112 ; 0,046816 ; 0,023408$;

0,$023408 ; 0,023408$

$$
\begin{aligned}
& =0,011704 \\
& y_{2}^{-}=\min
\end{aligned}
$$

0,$072760 ; 0,054570 ; 0,036380 ; 0,018190$; $\left\{\begin{array}{c}0,036380 ; 0,036380 ; 0,072760 ; 0,018190 ; \\ 0,054570 ; 0,036380\end{array}\right\}$

$$
\begin{gathered}
=0,018190 \\
y_{3}^{-}=\min
\end{gathered}
$$

$(0,017330 ; 0,017320 ; 0,051961 ; 0,051961 ;)$

$\{0,069282 ; 0,069282 ; 0,051961 ; 0,034641 ;\}$

$$
0,017320 ; 0,051961
$$

$$
\begin{aligned}
& =0,017320 \\
& y_{4}^{-}=\min
\end{aligned}
$$

0,$051634 ; 0,068824 ; 0,034412 ; 0,051618 ;$

$\left\{\begin{array}{c}0,068824 ; 0,017206 ; 0,034412 ; 0,034412 ; \\ 0,051618 ; 0,034412\end{array}\right\}$

$$
=0,017206
$$

$y_{5}^{-}=\min$ 
$(0,039392 ; 0,039392 ; 0,026261 ; 0,013131 ;$

$\left\{\begin{array}{c}0,052523 ; 0,013131 ; 0,026261 ; 0,013131 ; \\ 0,026261 ; 0,039392\end{array}\right\}$

$=0,013131$

$y_{6}^{-}=\min$

$(0,037796 ; 0,018898 ; 0,075593 ; 0,037796 ;)$

$\left\{\begin{array}{c}0,037796 ; 0,037796 ; 0,056695 ; 0,018898 ; \\ 0,075593 ; 0,037796\end{array}\right\}$

$=0,018898$

$y_{7}^{-}=\max$

$(0,083205 ; 0,055470 ; 0,055470 ; 0,083205 ;$

$\left\{\begin{array}{c}0,055470 ; 0,055470 ; 0,027735 ; 0,083205 ; \\ 0,055470 ; 0,055470\end{array}\right\}$

$=0,027735$

$A^{-}=(0,011704 ; 0,018190 ; 0,017321 ; 0,017206 ; 0,013131 ;$ 0,$018898 ; 0,027735$ )

Alternative distance Ai with positive ideal solution:

$$
D 1^{+}=
$$

$$
\begin{aligned}
& (0,035112-0,046816)^{2}+(0,072761-0,072761)^{2} \\
& (0,017321-0,069282)^{2}+(0,051619-0,068825)^{2} \\
& (0,039392-0,052523)^{2}+(0,037796-0,075593)^{2} \\
& \sqrt{(0,083205-0,083205)^{2}} \\
& =0,004734 \\
& D 2^{+}= \\
& (0,035112-0,046816)^{2}+(0,054571-0,072761)^{2} \\
& (0,017321-0,069282)^{2}+(0,068825-0,068825)^{2} \\
& (0,039392-0,052523)^{2}+(0,018898-0,075593)^{2} \\
& (0,055470-0,083205)^{2} \\
& =0,007324 \\
& D 3^{+}=
\end{aligned}
$$

$$
\begin{gathered}
(0,011704-0,046816)^{2}+(0,036380-0,072761)^{2} \\
(0,051962-0,069282)^{2}+(0,034412-0,068825)^{2} \\
(0,026261-0,052523)^{2}+(0,075593-0,075593)^{2} \\
(0,055470-0,083205)^{2} \\
=0,0055 \\
D 4^{+}=
\end{gathered}
$$

$$
\begin{gathered}
(0,011704-0,046816)^{2}+(0,018190-0,072761)^{2} \\
(0,051962-0,069282)^{2}+(0,051619-0,068825)^{2} \\
(0,013131-0,052523)^{2}+(0,037796-0,075593)^{2} \\
(0,083205-0,083205)^{2} \\
=0,007787 \\
D 5^{+}=
\end{gathered}
$$$$
(0,046816-0,046816)^{2}+(0,036380-0,072761)^{2}
$$$$
(0,069282-0,069282)^{2}+(0,068825-0,068825)^{2}
$$$$
(0,052523-0,052523)^{2}+(0,037796-0,075593)^{2}
$$$$
(0,055470-0,083205)^{2}
$$$$
=0,003658
$$$$
D 6^{+}=
$$

$$
\begin{gathered}
(0,035112-0,046816)^{2}+(0,036380-0,072761)^{2} \\
(0,069282-0,069282)^{2}+(0,017206-0,068825)^{2} \\
(0,013131-0,052523)^{2}+(0,037796-0,075593)^{2} \\
(0,055470-0,083205)^{2} \\
=0,007875
\end{gathered}
$$

$$
\begin{aligned}
& D 7^{+}= \\
& (0,046816-0,046816)^{2}+(0,072761-0,072761)^{2} \\
& (0,051962-0,069282)^{2}+(0,034412-0,068825)^{2} \\
& (0,026261-0,052523)^{2}+(0,056695-0,075593)^{2} \\
& (0,027735-0,083205)^{2} \\
& =0,005608 \\
& D 8^{+}= \\
& (0,023408-0,046816)^{2}+(0,018190-0,072761)^{2} \\
& (0,034641-0,069282)^{2}+(0,034412-0,068825)^{2} \\
& (0,013131-0,052523)^{2}+(0,018898-0,075593)^{2} \\
& (0,083205-0,083205)^{2} \\
& =0,010676 \\
& D 9^{+}= \\
& =0,005354 \\
& D 10^{+}= \\
& (0,023408-0,046816)^{2}+(0,036380-0,072761)^{2} \\
& (0,051962-0,069282)^{2}+(0,034412-0,068825)^{2} \\
& (0,039392-0,052523)^{2}+(0,037796-0,075593)^{2} \\
& (0,055470-0,083205)^{2} \\
& =0,005726
\end{aligned}
$$

Alternative distance Ai with negative ideal solution:

$$
D 1^{-}=
$$

$$
\begin{gathered}
(0,035112-0,011704)^{2}+(0,072760-0,018190)^{2} \\
(0,017321-0,017320)^{2}+(0,051619-0,017206)^{2} \\
(0,039392-0,013131)^{2}+(0,037796-0,018898)^{2} \\
(0,083205-0,027735)^{2} \\
=0,008834 \\
D 2^{-}=
\end{gathered}
$$

$$
\begin{aligned}
& (0,035112-0,011704)^{2}+(0,054571-0,018190)^{2} \\
& (0,017321-0,017320)^{2}+(0,068825-0,017206)^{2} \\
& (0,039392-0,013131)^{2}+(0,018898-0,018898)^{2} \\
& (0,055470-0,027735)^{2} \\
& =0,005995 \\
& D^{-}= \\
& \sqrt{\begin{array}{c}
(0,011704-0,011704)^{2}+(0,036380-0,018190)^{2} \\
(0,051962-0,017370)^{2}+(0,034412-0,017206)^{2} \\
(0,026261-0,013131)^{2}+(0,075593-0,018898)^{2} \\
(0,055470-0,027735)^{2} \\
=0,005983
\end{array}}
\end{aligned}
$$$$
\begin{gathered}
D 4^{-}= \\
\sqrt{\begin{array}{c}
(0,011704-0,011704)^{2}+(0,018190-0,018190)^{2} \\
(0,051962-0,017370)^{2}+(0,051619-0,017206)^{2} \\
(0,013131-0,013131)^{2}+(0,037796-0,018898)^{2} \\
(0,083205-0,027735)^{2}
\end{array}}
\end{gathered}
$$$$
=0,005818
$$ 
$D 5^{-}=$

$$
\begin{gathered}
(0,046816-0,011704)^{2}+(0,036380-0,018190)^{2} \\
(0,069282-0,017370)^{2}+(0,068825-0,017206)^{2} \\
(0,052523-0,013131)^{2}+(0,037796-0,018898)^{2} \\
(0,055470-0,027735)^{2} \\
=0,008921 \\
D 6^{-}=
\end{gathered}
$$$$
(0,035112-0,011704)^{2}+(0,036380-0,018190)^{2}
$$$$
(0,069282-0,017370)^{2}+(0,017206-0,017206)^{2}
$$$$
(0,013131-0,013131)^{2}+(0,037796-0,018898)^{2}
$$$$
(0,055470-0,027735)^{2}
$$$$
=0,004705
$$$$
D 7^{-}=
$$

$$
\begin{gathered}
(0,046816-0,011704)^{2}+(0,072761-0,018190)^{2} \\
(0,051962-0,017320)^{2}+(0,034412-0,017206)^{2} \\
(0,026261-0,013131)^{2}+(0,056695-0,018898)^{2} \\
(0,027735-0,027735)^{2} \\
=0,0073080 \\
D 8^{-}=
\end{gathered}
$$$$
(0,023408-0,011704)^{2}+(0,018190-0,018190)^{2}
$$$$
(0,034641-0,017320)^{2}+(0,034412-0,017206)^{2}
$$$$
(0,013131-0,013131)^{2}+(0,018898-0,018898)^{2}
$$$$
(0,083205-0,027735)^{2}
$$$$
=0,003810
$$$$
D 9^{-}=
$$$$
(0,023408-0,011704)^{2}+(0,055471-0,018190)^{2}
$$$$
(0,017321-0,017320)^{2}+(0,051619-0,017206)^{2}
$$$$
(0,026261-0,013131)^{2}+(0,075593-0,018898)^{2}
$$$$
(0,055470-0,027735)^{2}
$$$$
=0,006801
$$

$D 10^{-}=$

$(0,023408-0,011704)^{2}+(0,036380-0,018190)^{2}$

$(0,051962-0,017320)^{2}+(0,034412-0,017206)^{2}$

$(0,039392-0,013131)^{2}+(0,037796-0,018898)^{2}$

$$
(0,055470-0,027735)^{2}
$$

$$
=0,00378
$$

Determine preference score for each alternative:

$$
\begin{gathered}
V i=\frac{D_{I}^{-}}{D_{I}^{-}+D_{I}^{+}} \\
\mathrm{V} 1=\frac{0,008834}{0,008834+0,004734}=0,577350 \\
\mathrm{~V} 2=\frac{0,005995}{0,005995+0,007324}=0,474992 \\
\mathrm{~V} 3=\frac{0,005983}{0,005983+0,005500}=0,510529 \\
\mathrm{~V} 4=\frac{0,00518}{0,005818+0,007787}=0,463630 \\
\mathrm{~V} 5=\frac{0,008921}{0,008921+0,003658}=0,609622 \\
\mathrm{~V} 6=\frac{0,004705}{0,004705+0,007875}=0,435983 \\
\mathrm{~V} 7=\frac{0,007308}{0,007308+0,005608}=0,533048 \\
\mathrm{~V} 8=\frac{0,003810}{0,003810+0,010676}=0,373977 \\
\mathrm{~V} 9=\frac{0,006801}{0,006801+0,005354}=0,530333 \\
\mathrm{~V} 10=\frac{0,003780}{0,003780+0,005726}=0,448275
\end{gathered}
$$

\begin{tabular}{|c|c|c|c|c|c|c|c|c|}
\hline & $\mathrm{C} 1$ & $\mathrm{C} 2_{2}$ & $\mathrm{C} 3$ & $\mathrm{CA}_{4}$ & $C 5$ & $\mathrm{C6}$ & $\mathrm{C} 7$ & AKAR \\
\hline A1 & 3 & 4 & 1 & 3 & 3 & 2 & 3 & 2 \\
\hline A2 & 3 & 3 & 1 & 4 & 3 & 1 & 2 & 2 \\
\hline A3 & 1 & 2 & 3 & 2 & 2 & 4 & 2 & 2 \\
\hline A4 & 1 & 1 & 3 & 3 & 1 & 2 & 3 & 2 \\
\hline A5 & 4 & 2 & 4 & 4 & 4 & 2 & 2 & 2 \\
\hline A6 & 3 & 2 & 4 & 1 & 1 & 2 & 2 & 2 \\
\hline A7 & 4 & 4 & 3 & 2 & 2 & 3 & 1 & 2 \\
\hline A8 & 2 & 1 & 2 & 2 & 1 & 1 & 3 & 2 \\
\hline A9 & 2 & 3 & 1 & 3 & 2 & 4 & 2 & 2 \\
\hline A10 & 2 & 2 & 3 & 2 & 3 & 2 & 2 & 2 \\
\hline KUADRAT & 73 & 68 & 75 & 76 & 58 & 63 & 52 & \\
\hline AKAR & \begin{tabular}{|l|l|l|l|}
.544004 & 8. \\
\end{tabular} & \begin{tabular}{l|l|l|}
8.246211 & 8.6 \\
\end{tabular} & 8.660254 & 8.71779 & \begin{tabular}{c|c|}
798 & 7.61577 \\
\end{tabular} & \begin{tabular}{l|l|l}
773 & 7.93725 \\
\end{tabular} & $54 \quad 7.211103$ & \\
\hline \multicolumn{9}{|c|}{ Fig. 3. Matrix process } \\
\hline \multicolumn{9}{|c|}{ Normalize matrix results } \\
\hline & $\mathrm{x} 1$ & $\mathrm{X} 2$ & $\mathrm{X} 3$ & & $\mathrm{X} 4$ & $\times 5$ & $x_{6}$ & $\mathrm{X7}$ \\
\hline $\mathrm{X}_{1}$ & 0.351123 & \begin{tabular}{l|l}
3 & 0.485071 \\
\end{tabular} & 0.11 & \begin{tabular}{l|l}
1547 & 0. \\
\end{tabular} & 0.344124 & \begin{tabular}{|l|l|}
0.393919 \\
\end{tabular} & \begin{tabular}{|l|}
0.251976 \\
\end{tabular} & 0.416025 \\
\hline $\mathrm{X} 2$ & 0.351123 & \begin{tabular}{l|l}
3 & 0.363803 \\
\end{tabular} & & \begin{tabular}{l|l}
1547 & 0 \\
\end{tabular} & 0.458831 & \begin{tabular}{|l|l|}
0.393919 \\
\end{tabular} & \begin{tabular}{|l|l|l|l|l|} 
\\
\end{tabular} & 0.27735 \\
\hline$\times 3$ & 0.117041 & \begin{tabular}{l|l}
1 & 0.242536 \\
\end{tabular} & 0.34 & \begin{tabular}{l|l}
4641 & 0 \\
\end{tabular} & 0.229416 & 0.262613 & 0.503953 & 0.27735 \\
\hline$X_{4}$ & 0.117041 & \begin{tabular}{l|l}
1 & 0.121268 \\
\end{tabular} & 0.34 & \begin{tabular}{l|l}
4641 & 0 \\
\end{tabular} & 0.344124 & \begin{tabular}{|l|l|}
0.131306 \\
\end{tabular} & \begin{tabular}{|c|}
0.251976 \\
\end{tabular} & 0.416025 \\
\hline$\times 5$ & 0.351123 & \begin{tabular}{l|l}
3 & 0.242536 \\
\end{tabular} & & \begin{tabular}{l|l}
6188 & 0 \\
\end{tabular} & 0.458831 & 0.525226 & \begin{tabular}{|l|l|}
0.251976 \\
\end{tabular} & \begin{tabular}{|l|l|}
0.27735 \\
\end{tabular} \\
\hline$x_{6}$ & 0.351123 & \begin{tabular}{l|l}
3 & 0.242536 \\
\end{tabular} & & \begin{tabular}{l|l}
6188 & 0 \\
\end{tabular} & 0.114708 & \begin{tabular}{|l|l|}
0.131306 \\
\end{tabular} & \begin{tabular}{|l|}
0.251976 \\
\end{tabular} & 0.27735 \\
\hline$x 7$ & 0.468165 & \begin{tabular}{c|c|c|c|}
5 & 0.485071 \\
\end{tabular} & & \begin{tabular}{l|l}
4641 & 0 \\
\end{tabular} & 0.229416 & \begin{tabular}{|l|l|}
0.262613 \\
\end{tabular} & \begin{tabular}{|l|l|}
0.377964 \\
\end{tabular} & 0.138675 \\
\hline$x_{8}$ & 0.234082 & $\begin{array}{lll}2 & 0.121268\end{array}$ & & \begin{tabular}{l|l}
3094 & 0 \\
\end{tabular} & 0.229416 & 0.131306 & \begin{tabular}{|l|}
0.125988 \\
\end{tabular} & 0.416025 \\
\hline$\times 9$ & 0.234082 & \begin{tabular}{l|l|l|l|}
2 & 0.36380 \\
\end{tabular} & 0.11 & \begin{tabular}{l|l}
1547 & 0 \\
\end{tabular} & 0.344124 & \begin{tabular}{|l|l|}
0.262613 \\
\end{tabular} & \begin{tabular}{|l|}
0.503953 \\
\end{tabular} & \begin{tabular}{|l}
0.27735 \\
\end{tabular} \\
\hline$\times 10$ & 0.234082 & $\begin{array}{ll}2 & 0.242536 \\
\end{array}$ & & \begin{tabular}{ll|l}
4641 & 0 \\
\end{tabular} & 0.229416 & $\mid 0.393919$ & $|0.251976|$ & 0.27735 \\
\hline \multicolumn{9}{|c|}{$\begin{array}{l}\text { Fig. 4. Normalized matrix results } \\
\text { ge of each criterion }\end{array}$} \\
\hline 0,1 & 0,15 & $\mathbf{0 , 1 5}$ & $\mathbf{0 , 1}$ & 15 & $\mathbf{0 , 1}$ & 0,1 & 15 &, 2 \\
\hline
\end{tabular}

Published By:

\section{B. IMPLEMENTATION}

3. Normalize matrix results

4. Percentage of each criterion with criteria $(4,2,4,4,4,2,2)$ had the largest score and was the best solution to choose good qualified corn.

Decision support system application determined good qualified corn with using Microsoft Excel as follows: 1. Alternatives and criteria from run system.

\begin{tabular}{|c|c|c|c|c|c|c|c|}
\hline & $\mathrm{C} 1$ & $\mathrm{C} 2$ & $\mathrm{C} 3$ & $\mathrm{C} 4$ & $\mathrm{C} 5$ & $\mathrm{C} 6$ & $\mathrm{C7}$ \\
\hline $\mathrm{A} 1$ & 3 & 4 & 1 & 3 & 3 & 2 & 3 \\
\hline $\mathrm{A} 2$ & 3 & 3 & 1 & 4 & 3 & 1 & 2 \\
\hline $\mathrm{A} 3$ & 1 & 2 & 3 & 2 & 2 & 4 & 2 \\
\hline $\mathrm{A} 4$ & 1 & 1 & 3 & 3 & 1 & 2 & 3 \\
\hline A5 & 4 & 2 & 4 & 4 & 4 & 2 & 2 \\
\hline A6 & 3 & 2 & 4 & 1 & 1 & 2 & 2 \\
\hline A7 & 4 & 4 & 3 & 2 & 2 & 3 & 1 \\
\hline A8 & 2 & 1 & 2 & 2 & 1 & 1 & 3 \\
\hline A9 & 2 & 3 & 1 & 3 & 2 & 4 & 2 \\
\hline A10 & 2 & 2 & 3 & 2 & 3 & 2 & 2 \\
\hline
\end{tabular}

Fig. 2. Alternatives and criteria

2. Calculate normalized matrix decision $(\mathrm{R})$

Fig. 3. Matrix process

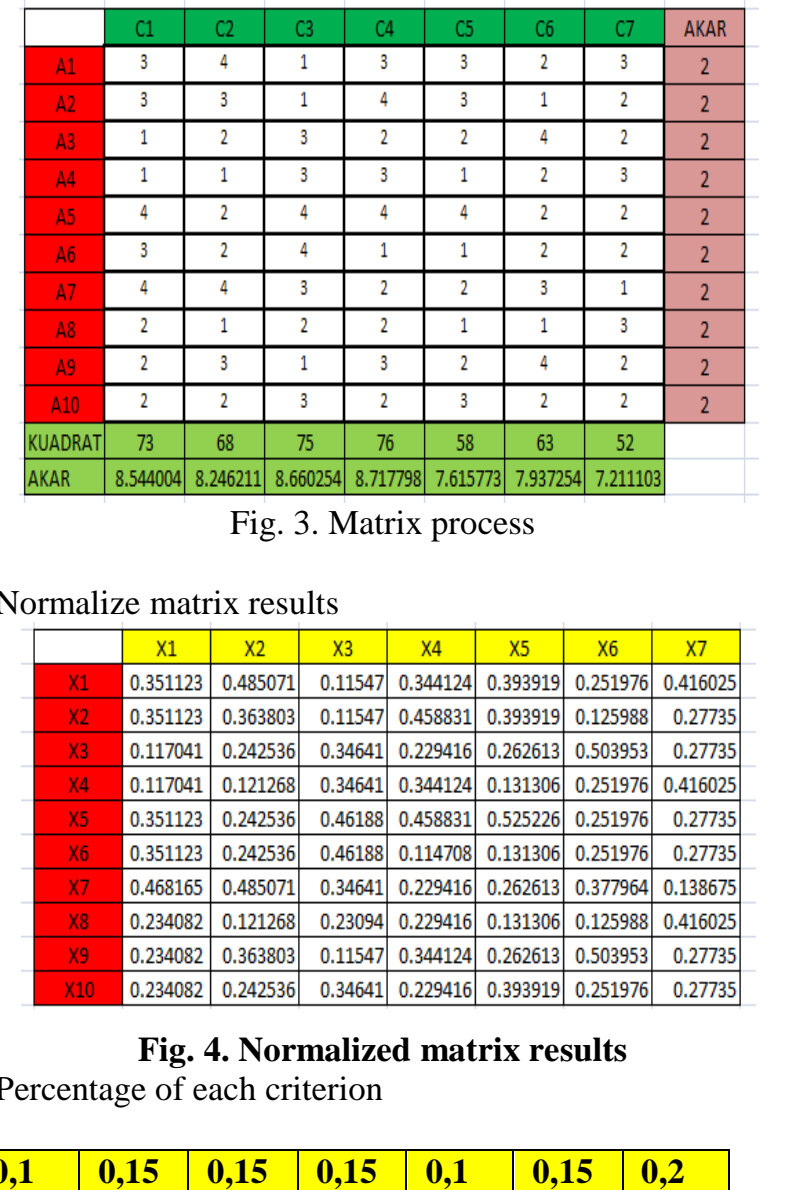

Fig. 4. Normalized matrix results Fig 4. Percentage 5. Y Matrix score 


\begin{tabular}{|c|c|c|c|c|c|c|c|}
\hline WJ & 0.1 & 0.15 & 0.15 & 0.15 & 0.1 & 0.15 & 0.2 \\
\hline V1 & 0.035112 & 0.072761 & 0.017321 & 0.051619 & 0.039392 & 0.037796 & 0.083205 \\
\hline V2 & 0.035112 & 0.054571 & 0.017321 & 0.068825 & 0.039392 & 0.018898 & 0.05547 \\
\hline V3 & 0.011704 & 0.03638 & 0.051962 & 0.034412 & 0.026261 & 0.075593 & 0.05547 \\
\hline V4 & 0.011704 & 0.01819 & 0.051962 & 0.051619 & 0.013131 & 0.037796 & 0.083205 \\
\hline V5 & 0.035112 & 0.03638 & 0.069282 & 0.068825 & 0.052523 & 0.037796 & 0.05547 \\
\hline V6 & 0.035112 & 0.03638 & 0.069282 & 0.017206 & 0.013131 & 0.037796 & 0.05547 \\
\hline V7 & 0.046816 & 0.072761 & 0.051962 & 0.034412 & 0.026261 & 0.056695 & 0.027735 \\
\hline V8 & 0.023408 & 0.01819 & 0.034641 & 0.034412 & 0.013131 & 0.018898 & 0.083205 \\
\hline V9 & 0.023408 & 0.054571 & 0.017321 & 0.051619 & 0.026261 & 0.075593 & 0.05547 \\
\hline V10 & 0.023408 & 0.03638 & 0.051962 & 0.034412 & 0.039392 & 0.037796 & 0.05547 \\
\hline & & & & & & &
\end{tabular}

Fig. 5. Y Matrix score

6. Calculate distance of ideal positive solution $(\mathrm{D}+)($ and ideal negative solution (D-)

\begin{tabular}{|c|c|c|c|c|}
\hline D & POSITIF & NEGATIF & AKAR + & AKAR - \\
\hline D1 & 0.004734 & 0.008834 & 0.068804 & 0.093988 \\
\hline D2 & 0.007324 & 0.005995 & 0.085579 & 0.077426 \\
\hline D3 & 0.0055 & 0.005983 & 0.074159 & 0.077349 \\
\hline D4 & 0.007787 & 0.005818 & 0.088245 & 0.076278 \\
\hline D5 & 0.003658 & 0.008921 & 0.060484 & 0.094453 \\
\hline D6 & 0.007875 & 0.004705 & 0.088738 & 0.068594 \\
\hline D7 & 0.005608 & 0.007308 & 0.074886 & 0.085486 \\
\hline D8 & 0.010676 & 0.00381 & 0.103325 & 0.061725 \\
\hline D9 & 0.005334 & 0.006801 & 0.073033 & 0.082466 \\
\hline 10 & 0.005726 & 0.00378 & 0.07567 & 0.061481 \\
\hline
\end{tabular}

Fig . 6. Ideal positive solution(D+) and ideal negative solution (D-)

7. Calculated preference score for each alternative

\begin{tabular}{|c|c|c|c|c|c|c|c|}
\hline D & POSITIF & NEGATIF & AKARt & AKAR - & $(t+1+-)$ & HASIL & RANKING \\
\hline 01 & 0.004734 & 0.008834 & 0.068804 & 0.093988 & 0.162793 & 0.57735 & 2 \\
\hline 02 & 0.007324 & 0.005995 & 0.085579 & 0.077426 & 0.163006 & 0.474992 & 6 \\
\hline D3 & 0.0055 & 0.005983 & 0.074159 & 0.077349 & 0.151508 & 0.510529 & 5 \\
\hline D4 & 0.007787 & 0.005818 & 0.088245 & 0.076278 & 0.164523 & 0.46363 & 7 \\
\hline 05 & 0.003658 & 0.008921 & 0.060484 & 0.094453 & 0.154937 & 0.609622 & 1 \\
\hline$D 6$ & 0.007875 & 0.004705 & 0.088738 & 0.068594 & 0.157333 & 0.435983 & 9 \\
\hline 07 & 0.005608 & 0.007308 & 0.074886 & 0.085486 & 0.160372 & 0.533048 & 3 \\
\hline 08 & 0.010676 & 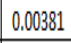 & 0.103325 & 0.061725 & 0.16505 & 0.373977 & 10 \\
\hline DS & 0.005334 & 0,006801 & 0.073033 & 0.082466 & 0.155499 & 0.530333 & 4 \\
\hline 10 & 0.005726 & 0.00378 & 0.07567 & 0.061481 & 0.137151 & 0.448275 & 8 \\
\hline
\end{tabular}

Fig. 7. Alternative preference

8. Ideal Matrix score (Max and Min)

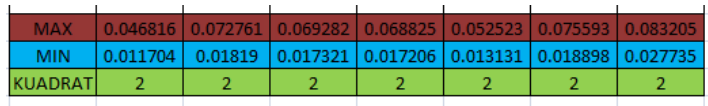

Fig. 8. Ideal Max, Min Matrix

\section{ANALYSIS RESEARCH RESULTS}

Testing results of the implementation of the TOPSIS method with the calculation manually, resulting rank in the weight of the criteria so obtained alternative 5 was chosen as good qualified corn with a score of V $5=0.609622$.

\section{CLOSING}

\section{A. Conclusion}

Decision support system using Technique For Order Preference By Similarity To Ideal Solution is a method that can help and facilitate in solving a problem, so it can help us in determining Good and qualified corn based on predefined criteria namely water content, deformed corn seed, corn waste, corn color, corn species, number of grains, corn cob. And using alternatives were tested then the alternative V5 with a score of 0.609622 was as good quality.

\section{Suggestion}

The following consists of some suggestions for further development of the following research:

1. The need to develop on other criteria so that the data obtained more accurately

2. In solving the multi criteria problem of the TOPSIS method is not the only decision making method that can be used, it is good to further study replace or compare with other methods such as AHP, WP etc.

3. Further research is suggested to make website-based applications more effective.

\section{REFERENCES}

1. N. P. Rahayu, R. Regasari, M. Putri, and A. W. Widodo, "Sistem Pendukung Keputusan ( SPK ) Pemilihan Tanaman Pangan Berdasarkan Kondisi Tanah Menggunakan Metode ELECTRE dan,” vol. 2, no. 8, 2018.

2. R. M. A. Adikara, M. T. Furqon, and A. Arwan, "Sistem Pendukung Keputusan Pemilihan Varietas Unggul Jagung Hibrida Menggunakan Metode AHP-SMART," vol. 2, no. 10, pp. 3373-3380, 2018.

3. N. Fitriyani and S. Ipnuwati, "Sistem Pendukung Keputusan Dalam Menentukan Ketua Osis Menggunakan Simple Additive Weighting ( SAW ) ( Studi Kasus : MTsN Model Talang Padang ) Jurusan Sistem Informasi STMIK Pringsewu Lampung," vol. 07, no. 01, pp. 414-415, 2016.

4. Y. P. P. Suyono1, RinaWati2, "Sistem Pendukung Keputusan Menentukan Kualitas Bibit Pala Menggunakan Metode SAW (Simple Additive Weighting) Suyono\#1,” vol. 07, no. 01, pp. 1-6, 2018.

5. O. K. Reflin Yadi1, Muhammad Sobri2, "Implementasi metode topsis untuk menentukan karyawan terbaik di pt.kfc cabang demang," pp. 1-9, 2015.

6. A. A. Chamid, "Prioritas Kondisi Rumah," vol. 7, no. 2, pp. 537-544, 2016.

7. H. Khair, "Respon Pertumbuhan Dan Produksi Tanaman Jagung (Zea mays L.) Terhadap Pemberian Pupuk Kandang Ayam Dan Pupuk Organik Cair Plus," vol. 18, no. 1, pp. 13-22, 2013.

8. S. Zulkifli, "Jurnal TAM ( Technology Acceptance Model ) Desember 2016 Decision Support System Pemberian Bonus Tahunan Pada Karyawan Berdasarkan Kinerja Karyawan Menggunakan Metode Simple Additive Weighting ( Study Kasus : STMIK PRINGSEWU)," vol. 7, no. 02, pp. 68-69, 2016.

9. T. S. dan S. F. Anggi Indah Yuliana 1*), "Pemupukan Bokashi DanCrotalaria juncea L .," vol. 1, no. 1, pp. 36-46, 2013.

10. T. Susilowati, A. Nazar, S. Mukodimah, M. Idris, F. Satria, P. Studi, S Informasi, P. Studi, M. Informatika, P. Studi, and M. Pendidikan, "Sistem pendukung keputusan penilaian kinerja guru sekolah dasar kecamatan gunung alip menggunakan metode topsis," vol. 9, no. 01, pp 37-38, 2018 . 